\title{
A Macrophage at Work
}

\section{Basil M. KAHWASH ${ }^{1}$, Samir KAHWASH ${ }^{2}$, Geok C. TAN ${ }^{2,3}$}

\footnotetext{
${ }^{1}$ Indiana University School of Medicine, Department of Internal Medicine, Indianapolis IN, USA

${ }^{2}$ Nationwide Children's Hospital, Department of Pathology and Laboratory Medicine, Columbus OH, USA

${ }^{3}$ National University of Malaysia, Department of Pathology, Kuala Lumpur, MALAYSIA
}

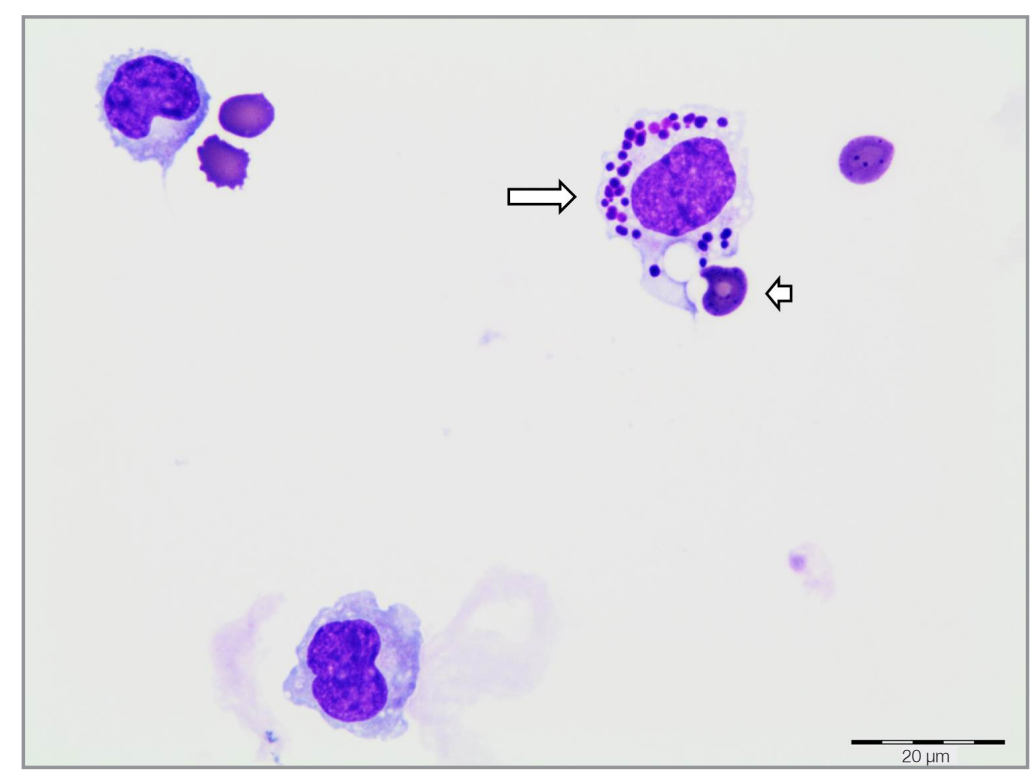

In this image a macrophage in cerebrospinal fluid (CSF) is caught doing what it does best; cleansing the environment of objects that don't belong. The patient, a 6-month-old girl, initially presented with choking and feeding difficulty. Brain MRI revealed a large tumour in the right posterior fossa, originating from the inferior aspect of the right cerebellar hemisphere. She underwent surgery to remove the tumour a few days before the CSF was sampled. Histologic examination confirmed the diagnosis of Atypical Teratoid Rhabdoid Tumor.

The macrophage is seen stretching its pseudopods -like a well-trained athlete reaching out to make a catch- to grab a red blood cell (RBC) (short arrow). Particles of digested debris from other RBCs are noted within its cytoplasm (long arrow) - evidence that this phagocytic act had been performed several times before. Macrophages, via chemotaxis, play a significant role in cleansing surgical sites by removing debris and organisms.

\section{Correspondence:}

Geok Chin TAN

Department of Pathology and Laboratory Medicine

Nationwide Children's Hospital

Columbus OH 43205 / USA

Tel: $+1-614-722-5422$

Fax: +1-614-722-2899

e-mail: geokchin.tan@nationwidechildrens.org 